\title{
Temporal Pointing Variations of The Solar Dynamics Observatory's HMI and AIA Instruments on Sub-Weekly Time Scales
}

\author{
N. Brice Orange, Hakeem M. Oluseyi, David \\ L. Chesny, Maulik Patel, Patrick Champey, \\ Katie Hesterly, Dylan Anthony, and Robert \\ Treen
}

(C) Springer $\bullet \bullet \bullet \bullet$

\begin{abstract}
Achieving sub-arcsecond co-registration across varying time-lines of multi-wavelength and instrument images is not trivial, and requires accurate characterization of instrument pointing jitter. In this work we have investigated internal pointing errors, on daily and yearly time-scales, occurring across the Solar Dynamics Observatory's (SDO) Atmospheric Imaging Assembly (AIA) and Helioseismic Magnetic Imager (HMI). Using cross-correlation techniques on AIA $1700 \AA$ passband and HMI line-of-sight (LOS) magnetograms, from three years of observational image pairs at approximately three day intervals, internal pointing errors are quantified. Pointing variations of $\pm 0.26^{\prime \prime}$ (jitter limited) and $\pm 0.50^{\prime \prime}$ in the solar East-West $(x)$ and North-South $(y)$ directions, respectively, are measured. AIA observations of the Venus June 2012 transit are used to measure existing coalignment offsets in all passbands. We find AIA passband pointing variations are $\left\langle\Delta X_{C O}\right\rangle=1.10^{\prime \prime} \pm 1.41^{\prime \prime}$ and $\left\langle\Delta Y_{C O}\right\rangle=1.25^{\prime \prime} \pm 1.24^{\prime \prime}$, when aligned to HMI's nominal image center, referred to herein as the CutOut technique $(\mathrm{CO})$. Minimal long-term pointing variations found between limb and correlation derived pointings provide evidence that image center positions provided by the instrument teams achieve single pixel accuracy on time-scales below their characterization. However, daily AIA passband pointing variations of $\lesssim 1.18^{\prime \prime}$ indicate autonomous sub-arcsecond co-registration is not yet fully achievable.
\end{abstract}

\section{Introduction}

Addressing the coronal heating problem is not trivial, as it requires the use of high resolution multi-wavelength and temporal images with sub-arcsecond co-registration. Currently, in the solar physics field the Solar Dynamics Observatory's (SDO) Atmospheric Imaging Assembly (AIA; Lemen et al. 2012)

Department of Physics \& Space Sciences, Florida Institute of Technology, Melbourne, FL 32901, USA 
and Heliographic Magnetic Imager (HMI; Schou et al., 2012) instruments are providing unprecedented amounts, approximately one image a second, of high resolution data $\left(\lesssim 0.6^{\prime \prime}\right)$. AIA takes full-disk images of the Sun in the following nine passbands: $94 \AA(\log T \approx 6.8), 131 \AA(\log T \approx 5.8), 171 \AA(\log T \approx 5.9), 193 \AA$ $(\log T \approx 6.2,7.2), 211 \AA(\log T \approx 6.3), 304 \AA(\log T \approx 4.8), 335 \AA(\log T \approx 6.4)$, $1600 \AA(\log T \approx 5.0), 1700 \AA(\log T \approx 3.7)$, and $4500 \AA(\log T \approx 3.7$; observed typically every $\approx 30 \mathrm{~min}$ ), while HMI takes full-disk images of the Sun's line-of-sight (LOS) magnetic field $(\approx 45 \mathrm{~s})$.

Some recent studies have highlighted that commonly used multi-passband alignments for AIA and HMI, considered to yield sub-pixel $\left(\leq 0.6^{\prime \prime}\right)$ accuracy, deliver a less than optimized status $\left(\approx 1^{\prime \prime}\right.$; Del Zanna, O'Dwyer, and Mason, 2011 . Brooks, Warren, and Ugarte-Urra, 2012). These alignment techniques, both of which utilize aia_prep.pro, i.e., standard Solar SoftWare (SSW), and result in level-1.5 data, are summarized as follows: aligning multi-passband chargecouple device (CCD) centers as Sun center (SC) or using a single passband's documented alignment information as a fiducial reference position for all other passbands. Thermal jitter motion, which places a systematic limit on achievable co-alignment accuracy, reported to affect each AIA telescope is $\approx 0.3^{\prime \prime}$ (Lemen et al., 2012, Aschwanden et al., 2013), while that of HMI remains un-reported (as of time of writing; Schou et al. |2012).

Shine et al. (2011) showed AIA passband image pointings are not temporally stable, suggested as a result of thermal flexing, for the four AIA telescopes and indicated monitoring and characterization of each telescope's nominal pointing (i.e., offset relative to solar center) is required. Currently, per AIA passband, image pointings are defined from an automated limb finder routine that performs an interactive Hough transform on candidate limb pixels to converge on the solar center $(x, y)$ and define it's radial distance (Shine et al., 2011). A daily average of these image center positions, performed once a week, are used to define the master pointings that are propagated in documented header information (Shine et al., 2011). However, image positions change significantly on weekly time scales, and daily variations $\left(\gtrsim 0.6^{\prime \prime}\right)$ are not characterized by master pointings (Shine et al., 2011). AIA image plate scales and angles are considered significantly more stable than that of the aforementioned offsets, with Shine et al. (2011) indicating no evidence of scale change and angle variations of $0.001^{\circ}$. HMI's master pointings are defined from similar techniques and time scales as those used by AIA.

Using cross-correlation techniques on AIA and HMI observational image pairs, performed on sub-weekly time-scales, we measure instrument jitter in the master pointing data by directly comparing limb and correlated image center positions. The June 2012 Venus transit is used to measure pointing variations of each of AIA's passbands. Throughout the remainder of this paper the term SC technique refers to multi-passband image CCD centers defined as Sun center, while CutOut is that where HMI's master pointing is as the fiducial reference position for all AIA passbands. It is noted, reported co-registration discrepancies are consistent across both co-alignment techniques summarized above, but result in a constant solar center offset $\approx 5^{\prime \prime}$ (Figure 1) between the two (Boerner 2013, private communication). 
Table 1. Observational date range, image time difference $(\langle\Delta t\rangle$; in seconds), typical observed $x$ and $y$ image center offsets $\left(\left\langle R_{x}^{\text {obs }}\right\rangle\right.$ and $\left\langle R_{y}^{\text {obs }}\right\rangle$, respectively, in arcsec) for co-aligned $1700 \AA$ far-ultraviolet (FUV) images and LOS magnetograms.

\begin{tabular}{cccc}
\hline Obs. Date Range & $\langle\Delta t\rangle$ & $\left\langle R_{x}^{\text {obs }}\right\rangle$ & $\left\langle R_{y}^{\text {obs }}\right\rangle$ \\
\hline May 2010 - March 2013 & 5.71 & 0.26 & 0.50 \\
\hline
\end{tabular}

In that respect, the remainder of this paper is outlined as follows: in Section 2 we provide a long-term, sub-weekly, characterization of AIA and HMI instrument jitter and compare image pointings resultant from limb fitting and cross-correlation techniques; Section 3 quantifies AIA passband pointing variations via observational data from the June 2012 Venus transit; a discussion of our findings is provided in Section 4 and concluding remarks are given in Section 5.

\section{AIA and HMI Sub-Weekly Pointing Errors}

Measurements of AIA and HMI's instrument pointing variations are performed at approximately three day intervals spanning May 2010-March 2013 from cross-correlated image pairs of the $1700 \AA$ passband (which directly observes the solar photosphere) and LOS magnetograms. Cross-correlation, widely considered to yield sufficient accuracy $(\lesssim 1$ pixel; e.g., Brooks, Warren, and Ugarte-Urra 2012, Del Zanna, O'Dwyer, and Mason 2011) is done using visually bright point-like features or magnetic neutral lines (Figure 1).

Image pairs were pre-processed to level-1.5 using aia_prep.pro (which included roll angle corrections), CutOut aligned, and magnetograms interpolated to the effective resolution of FUV images $\left(\approx 0.6^{\prime \prime}\right)$. Solar rotation effects, $\approx 0.17$ arcsec $\min ^{-1}$ (Sarro and Berihuete, 2011) close to disk center, were minimized by using observational time differences below AIA's thermal jitter motion (i.e., $\leq 2 \mathrm{~min}$ ), and the standard SSW routine drot_map.pro. FUV and magnetogram images were then mapped to solar coordinates by calibrating each image's axes with respect to actual Stonyhurst heliographic coordinates, i.e, coordinate $(0,0)$ corresponds to solar center. Disk regions of $\pm 300^{\prime \prime}$ from solar center are only considered to minimize projection effects. Per observational image pair, two measurements of the $x$ and $y$ co-alignment offsets, relative to HMI's master pointings ( $R_{x}^{\text {obs }}$ and $R_{y}^{\text {obs }}$, respectively), were made on regions comprised of bright network and small compact features (e.g., similar to those observed in Figure 1. The resultant $R_{x}^{\text {obs }}$ and $R_{y}^{\text {obs }}$ offsets were then smoothed per day with errors propagated from the differences in daily measurements.

Figure 2 displays AIA and HMI's master pointings (i.e., $X C / Y C_{\mathrm{mp}}$ derived from limb fitting techniques) compared to the pointings obtained from crosscorrelating image pairs (i.e., $X C / Y C_{\text {obs }}$ ) at sub-weekly time-scales. A summary of our co-alignment offsets are provided in Table 1 . with typical values of $\approx 0.3^{\prime \prime}$ 

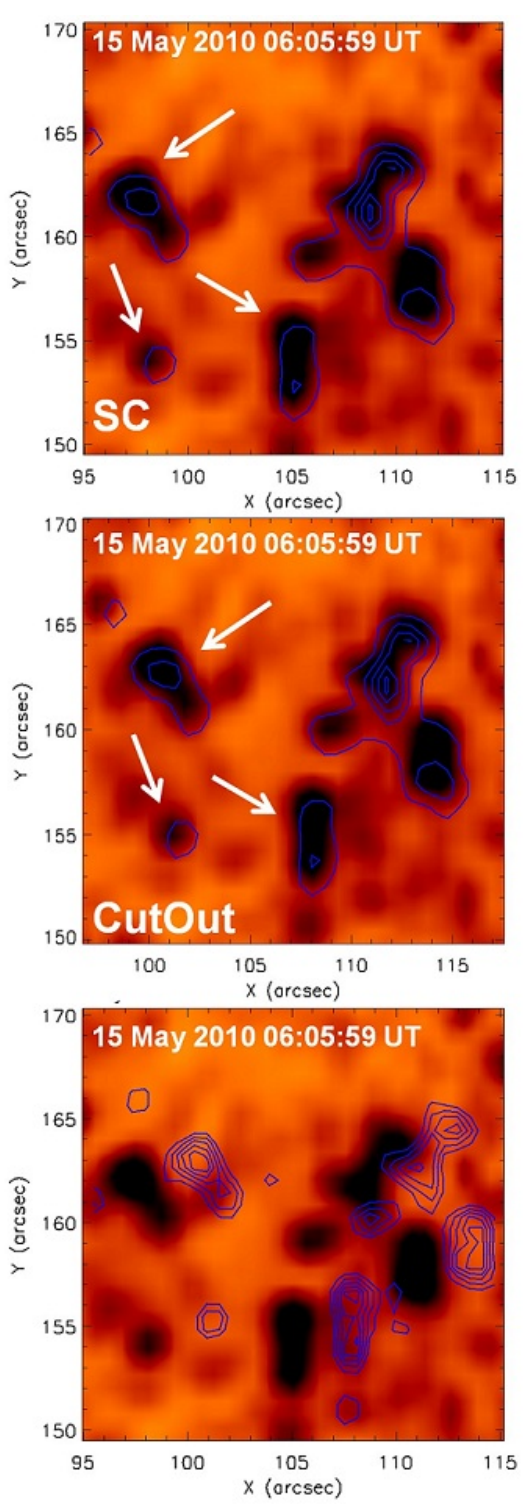

Figure 1. The top and middle panels (pre-processed under SC and CutOut techniques, respectively) are AIA $1700 \AA$ intensity images $\left(\mathrm{DN} \mathrm{pix}{ }^{-1} \mathrm{~s}^{-1}\right)$, shown on inverted color scale, with contours showing regions of positive magnetic flux (blue solid lines) derived from HMI's LOS magnetograms and plotted at levels of $\pm 25-100 \mathrm{G}$. Bottom panel is AIA $1700 \AA$ same as that in the top but contours are from the middle panel image, which shows the difference between the pre-processing techniques is a solar center offset $\left(\approx 5^{\prime \prime}\right.$ in both the $x$ and $y$ directions).

and $0.5^{\prime \prime}$ (in solar $x$ and $y$ directions, respectively). These results are consistent with reported mis-alignments $\left(\lesssim 1^{\prime \prime}\right.$; Del Zanna, O'Dwyer, and Mason, 2011; Brooks, Warren, and Ugarte-Urra, 2012) as well as those expected from daily 

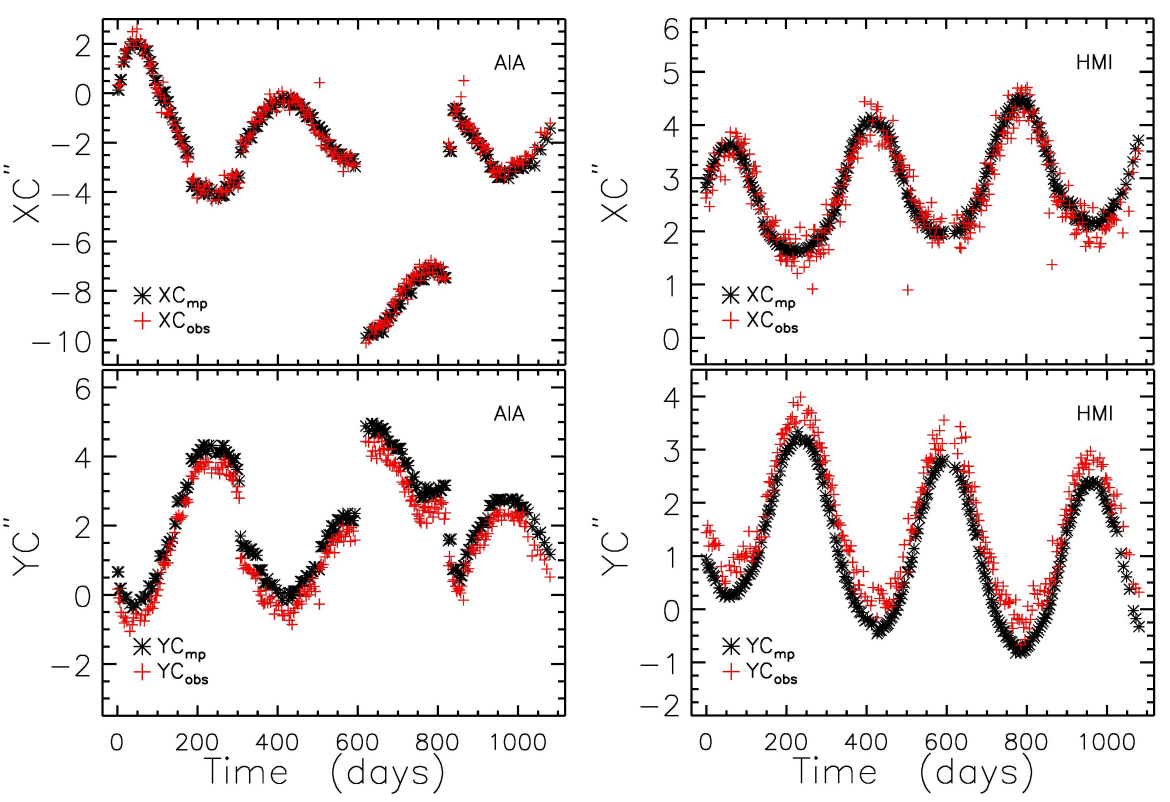

Figure 2. Top, from left to right, are plots of AIA and HMI master pointings, respectively, $\left(X C_{\mathrm{mp}}\right.$; asterisks) provided by the SDO teams and observed image centers from cross-correlation techniques ( $X C_{\text {obs }}$; red pluses) in the solar $x$ direction versus time (May 2010-March 2013), respectively. Bottom, similar to top panels for pointings in the solar $y$ direction.

variations $\left(\lesssim 1.2^{\prime \prime}\right.$; Shine et al. 2011$)$. Moreover, $R_{x}^{\text {obs }}$ is within the limits of instrumental jitter, and as observed in Figure 2 is indicative of random scatter. Solar $y$ pointing offsets varied minimally $\left(\approx \pm 0.02^{\prime \prime}\right)$ over the three year span, and indicate their alignment agreement could be autonomously improved (Figure 2).

\section{Using the Venus Transit for Investigation of Internal AIA Co-Registration Errors}

Investigation of daily AIA pointing variations uses observational data of the 5-6 June 2012 Venus transit (23:00:00 UT-04:00:00 UT), while Venus' silhouette was fully visible on the solar disk (Figure 3). Venus' transit provides the ideal fiducial reference for measuring such errors since its silhouette must be seen at identical locations in each passband when the transit speed across the solar surface is accounted for (Kamio et al., 2010; Shimizu et al., 2007).

Daily pointing variations from the various AIA passbands are determined from a direct comparison of the heliographic coordinates of Venus' center to those predicted by deriving the Venus' velocity using running difference images $(1700 \AA$ passband; see Figure 44. The details of our observational data and method as well as results are described in the next two Sections 3.1 and 3.2. 


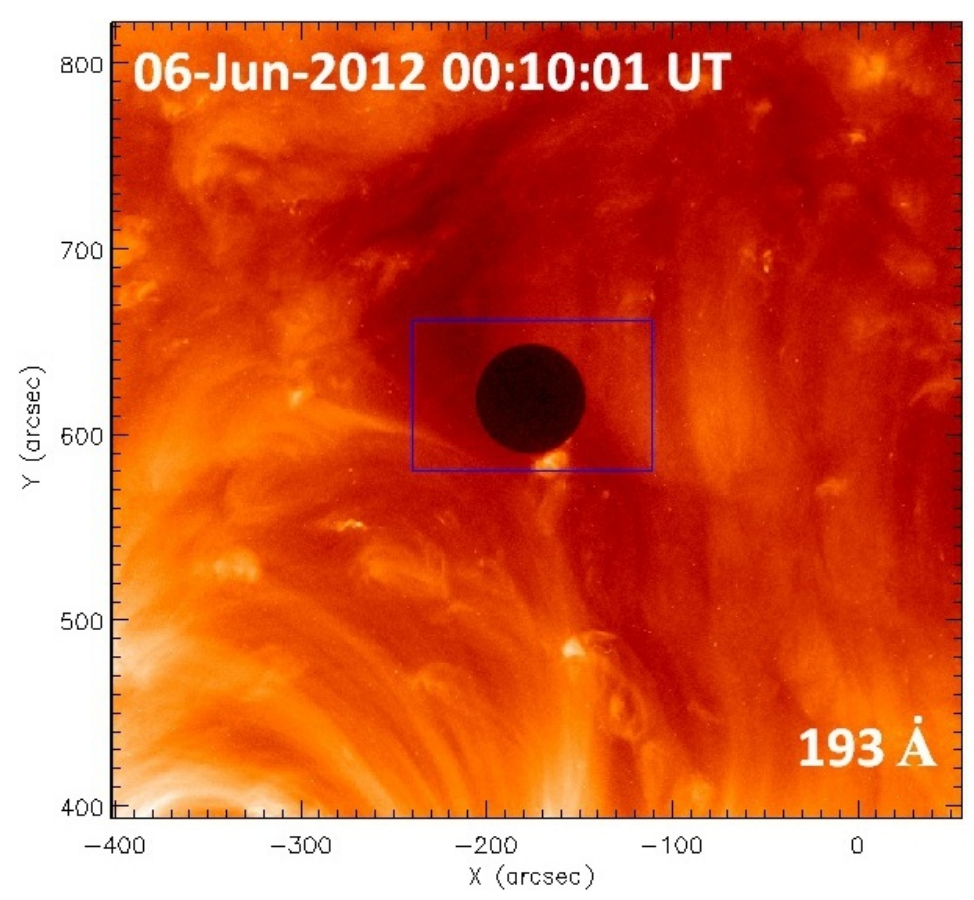

Figure 3. AIA $193 \AA$ passband observed on 6 June 2012 at 00:10:01 UT. The image is displayed on a $\log _{10}$ scale and the boxed blue region surrounds the disk of Venus.

\subsection{Venus Transit Analysis}

The Venus transit data were pre-processed to obtain both SC and Cut-Out multiimage alignments, as well as those aligned to their respective master pointings (MP). For each pre-processing technique, a ten minute temporal cadence was used which resulted in $\approx 30$ images of each passband with exception of $4500 \AA$ images (5 observations). Custom written software, discussed in depth below, was then used to measure the heliographic coordinates of Venus' disk centroid from all images.

The software measured individually the vertical, North - South (N-S), and horizontal, East - West (E-W), disk centroid positions vi a calculating and comparing observed disk diameters to the planet's known angular size $\left(58.3^{\prime \prime}\right.$; Odenwald, 2012). Calculation of the disk diameter required user selection of loci regions corresponding to a single orientation's limb position (e.g., leading and trailing edges for $\mathrm{E}-\mathrm{W}$, see Figure 5p. Per loci region, limb positions along the vertical or horizontal for $\mathrm{E}-\mathrm{W}$ and $\mathrm{N}-\mathrm{S}$ diameters, respectively, were determined by measuring where the steepest flux gradients occurred (Figure 55). The position of steepest flux gradient occurring furthest away from disk center was stored and visually checked. Uncertainties in each of the aforementioned measurements were derived from the plate scale of a single pixel per passband (defined in the header of the FITS data file) and residuals from comparing measured angular sizes to the known value. 




Figure 4. Sequence of AIA $1700 \AA$ images, observed on 5-6 June 2012 from 23:00:07 UT - 03:52:07 UT (left to right, respectively) with pluses denoting Venus' centroid, defined by the technique discussed in the text (Section 3.1. Arrows are shown to represent the running difference technique applied to the data to measure the planet's transit velocity in the $x$ and $y$ directions and provide fiducial references as a function of time from which co-alignment discrepancies between all other AIA passbands are measured. 


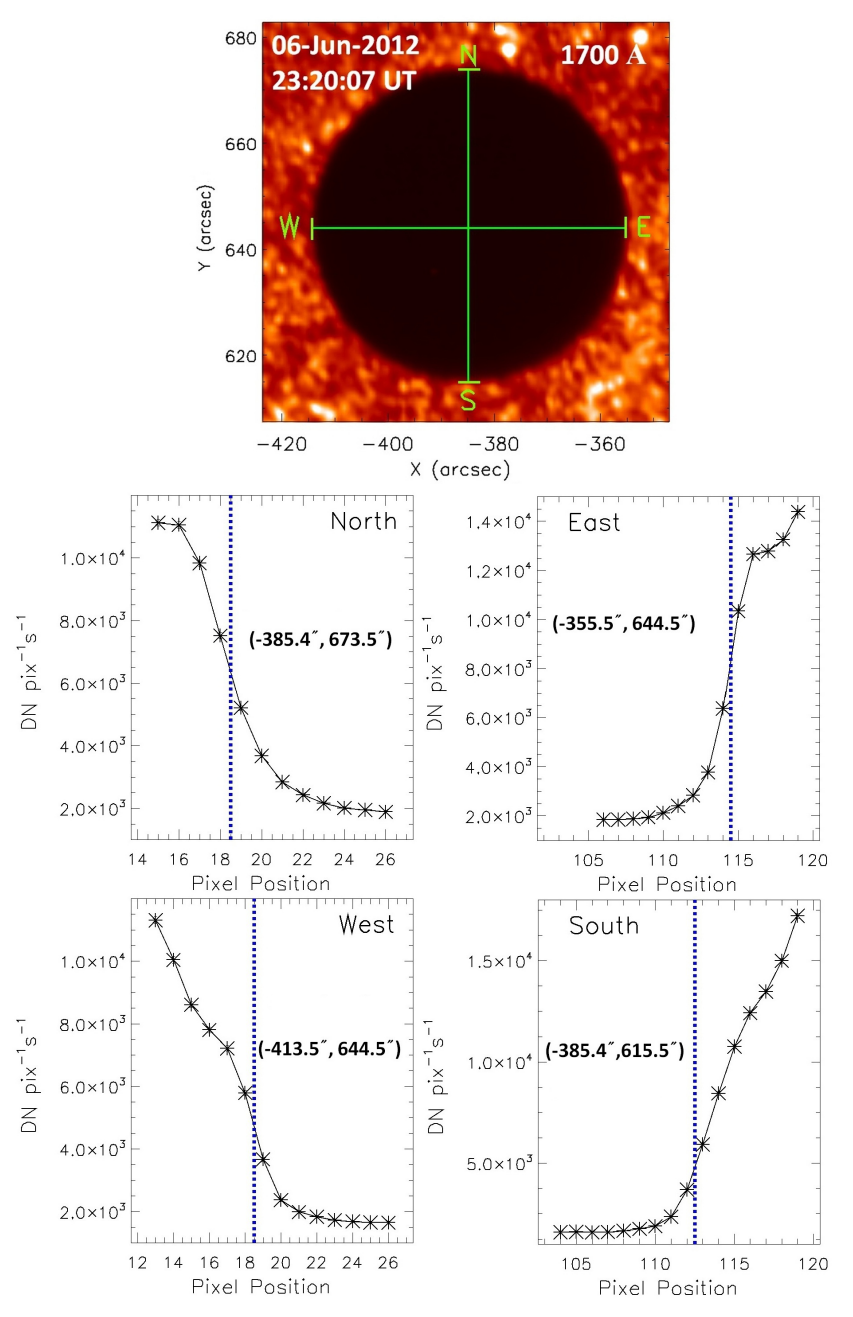

Figure 5. The top panel is an intensity image centered on the silhouette of Venus's disk from AIA's 1700 ̊̊ passband observed on 5 June 2012 23:20:07 UT, with the center of the crosshairs representing the measured center of Venus' disk. The four bottom panels are flux versus pixel position for the North, East, South, and West positions noted in the top panel from top left to bottom left in clockwise direction, respectively. Note, vertical dotted blue lines denote measured limb positions, as outlined in the text, of $\left(-385.4^{\prime \prime}, 673.5^{\prime \prime}\right),\left(-385.4^{\prime \prime}, 673.5^{\prime \prime}\right),\left(-355.5^{\prime \prime}, 644.5^{\prime \prime}\right)$, and $\left(-413.5^{\prime \prime}, 644.5^{\prime \prime}\right)$ for the North, East, South, and West positions, respectively, presented here in the form of solar $x$ and $y$ directions, respectively.

Figure 5 provides an example of this entire process. Its left panel displays the resultant diameters (solid lines, plotted with a width of $\approx 1^{\prime \prime}$ to be easily observable) and limb positions (marked by their respective direction and a solid line). The right panel displays flux versus position for the $\mathrm{N}-\mathrm{S}$ and $\mathrm{E}-\mathrm{W}$ disk edges corresponding to the aforementioned marked locations on the intensity 

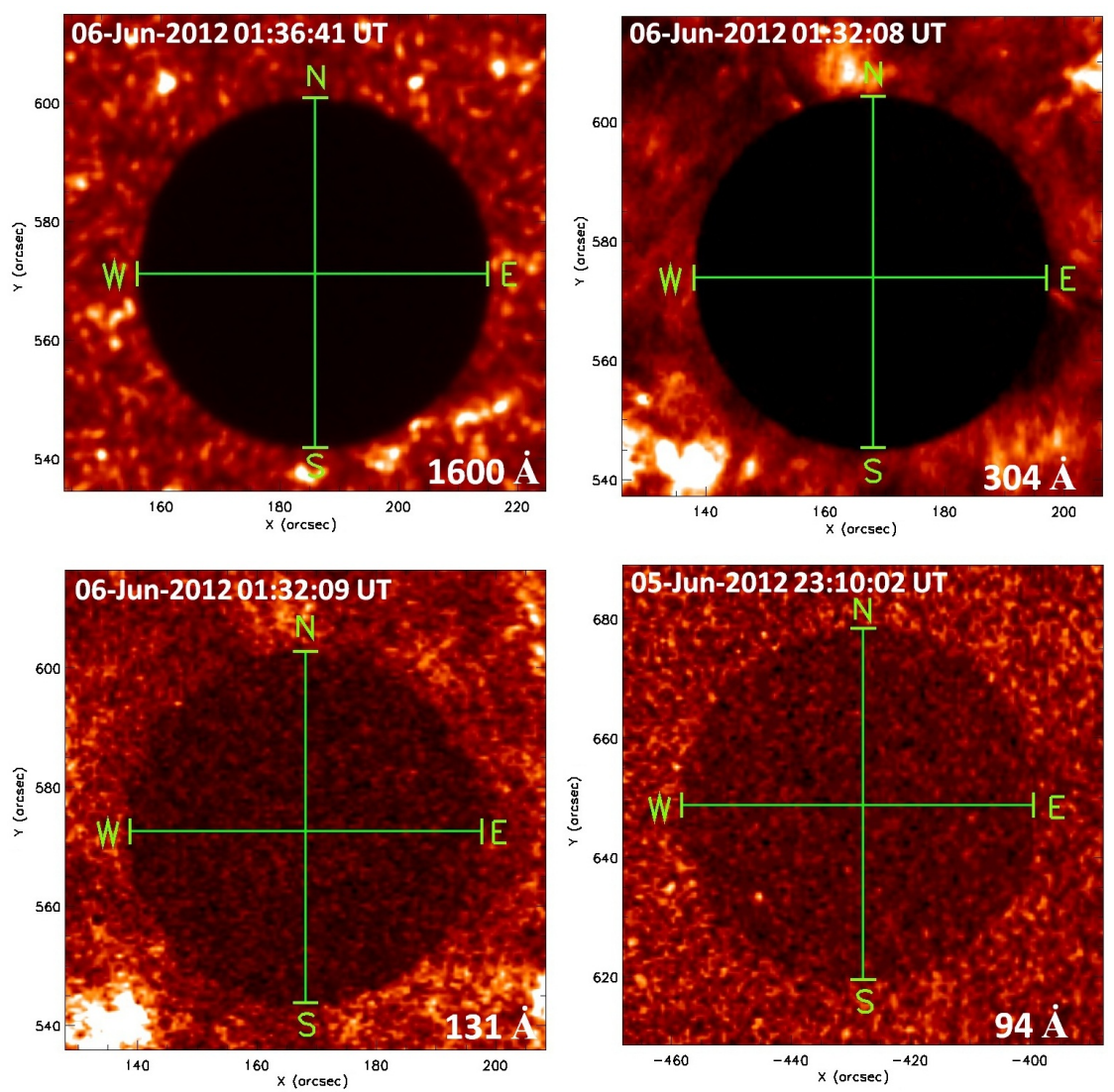

Figure 6. Intensity images centered on the silhouette of Venus' disk from AIA's $1600 \AA, 304 \AA$, $94 \AA$, and $131 \AA$ passbands from top right to bottom right in clockwise direction, respectively, observed on 5-6 June 2012 showing variations in the contrast of Venus' disk as observed by different passbands. On each image the center of the crosshairs represents Venus' measured centers determined from the techniques discussed in the text, respectively.

image of the right panel. Figure 6 has also been provided to illustrate the variations in Venus' contrast against the solar disk for a sample of passbands. It is noted, features which show up on Venus' disk in $131 \AA$ and $94 \AA$ images of Figure 6 are directly indicative of the stray light which affects all AIA EUV passbands (Poduval et al., 2013).

Using running differences of Venus' disk center position, its transit velocity relative to the solar surface was measured to be $v_{x}=6.7 \times 10^{-2} \pm 6.3 \times 10^{-4}$ arcsec $\mathrm{s}^{-1}$ and $v_{y}=-9.2 \times 10^{-3} \pm 6.3 \times 10^{-4}$ arcsec $\mathrm{s}^{-1}$ in the horizontal and vertical directions, respectively (Figure 4). Observational disk centers and the transit speed were then used to generate a set of predicted disk positions at one second intervals over the observational time frame used. Co-alignment offsets $(\Delta X$, $\Delta Y$ ), relative to $1700 \AA$ images as function of transit time, were measured for co-aligned multi-passband data. 
Table 2. Typical co-alignment offsets, in arcsec, (averaged over seven AIA passbands, and measured relative to the $1700 \AA$ image positions) and their resultant uncertainties, defined as the standard error on the mean (SEM), for pre-processing images under SC, MP, and CutOut assumptions.

\begin{tabular}{ccccc}
\hline Pre-Process & $\langle X\rangle$ & $\sigma_{S E M}^{X}$ & $\langle Y\rangle$ & $\sigma_{S E M}^{Y}$ \\
\hline SC & 1.47 & 2.86 & 1.83 & 2.29 \\
MP & 1.29 & 1.60 & 1.37 & 1.66 \\
Cut-Out & 0.87 & 1.14 & 1.18 & 1.46 \\
\hline
\end{tabular}

\subsection{Results}

Figures 7 and 8 provide the $x$ and $y$ co-alignment offsets, respectively, (plotted at $\approx 30$ min intervals for clarity) of AIA passbands imaged by telescopes 1,2 , and 4 (Lemen et al., 2012). Figure 9 displays both the $x$ and $y$ co-alignment offsets of AIA passbands imaged by telescope 3 (Lemen et al., 2012). As observed in these figures, significant variance exists, not only between disk center positions but as a function of time, too. Most typically, the $x$ and $y$ pointing variations are indicative of random scatter, but a number of passbands do exhibit a quasiperiodic nature in the $x$ direction. Using the $304 \AA$ passband the period of this variation was estimated to be $\approx 5 \mathrm{~h}$. However, we note that it is only a crude approximation since variations are found in other passbands $(\approx \pm 30 \mathrm{~min}$, Figures 7 and 9 while our observation sequence did not cover a full period.

Table 2 presents the co-alignment offsets, averaged over seven passbands, and uncertainties, defined as the standard error on the mean (SEM), for each preprocessing technique. It is noted, these results are not weighted by measurements derived from $94 \AA$ and $4500 \AA$ images for the following reasons: the low countrates of the $94 \AA$ passband (Boerner et al., 2012), and the significantly longer temporal cadence of the $4500 \AA$ passband. The results of Table 2 indicate that image pointings vary by $\gtrsim 2$ pixel, independent of solar direction, on daily timescales. We point out, no technique results in sub-pixel $\left(\lesssim 0.6^{\prime \prime}\right)$ alignment, as expected (Shine et al., 2011), while resultant uncertainties indicate minimal variations of $\approx 1.3^{\prime \prime}$ occur over a few hours.

Co-alignment offsets per passband were re-measured by letting individual passbands serve as the fiducial reference (i.e., using running image differences to derive and predict the planet's disk center positions), again barring $94 \AA$ and $4500 \AA$ images. The results of this analysis are shown in Table 3 . As observed, the typical alignment accuracy, as well as its respective uncertainty, is consistent with our previous reports for the $1700 \AA$ passband. Therefore, using our resultant co-alignment offsets, the typical AIA passband alignment accuracy, derived from eight passbands, is

$$
\begin{aligned}
& \left\langle\Delta X_{C O}\right\rangle=1 .^{\prime \prime} 10 \pm 1 .^{\prime \prime} 41 \text { and } \\
& \left\langle\Delta Y_{C O}\right\rangle=1 .^{\prime \prime} 25 \pm 1 .^{\prime \prime} 24
\end{aligned}
$$




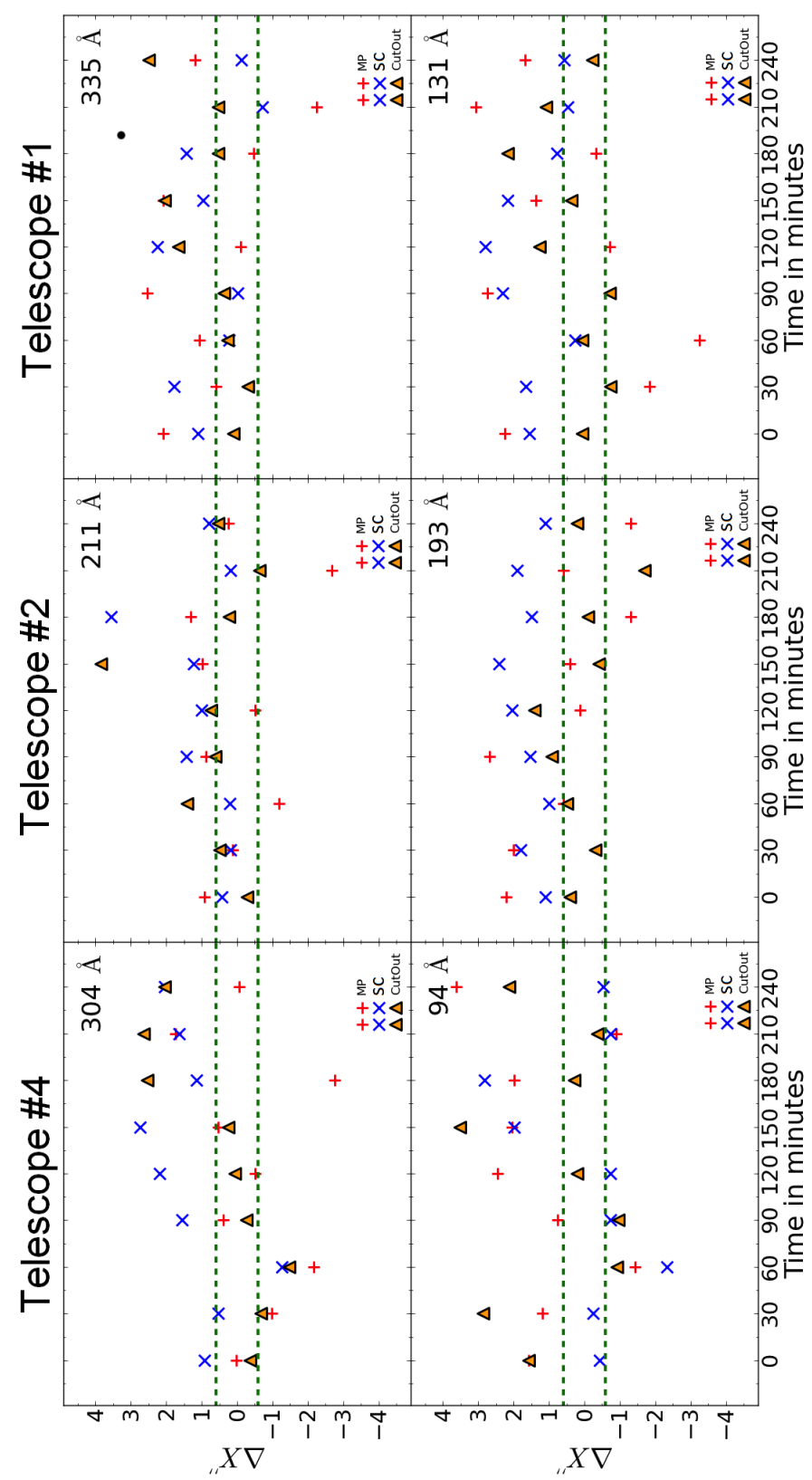

Figure 7. Solar $x$ co-alignment offsets $(\Delta X)$, relative to $1700 \AA$ image positions, versus time (minutes measured relative to 5 June 2012 23:00:00 UT) shown as a function of AIA telescope number (1, 2, and 4 only; Lemen et al., 2012) and smoothed over $\approx 30 \mathrm{~min}$ for visual clarity. Pre-processing techniques of MP, SC and CutOut are represented by red pluses, blue $\mathrm{x}$-signs, and gold triangles on each plot, respectively. 


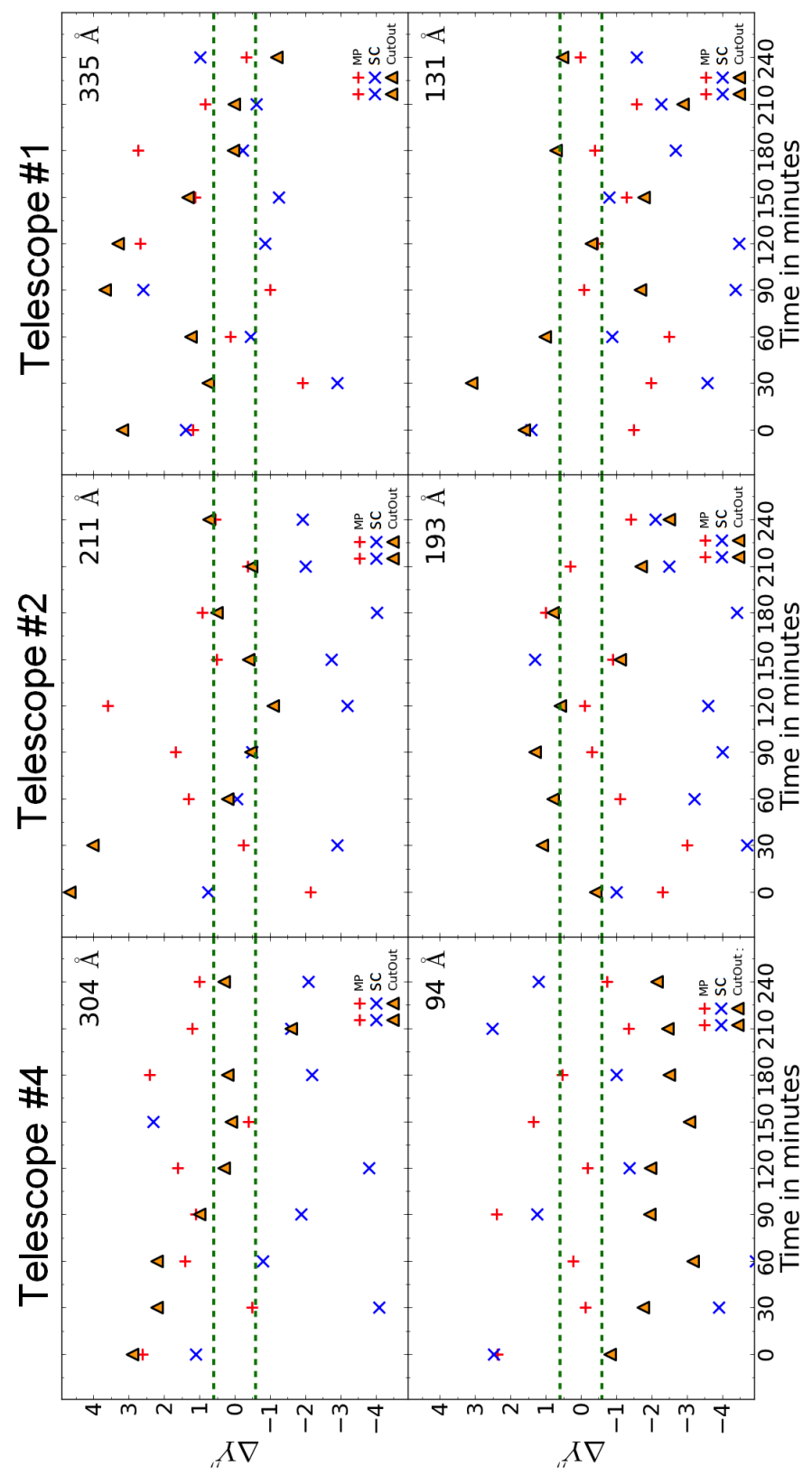

Figure 8. Solar $y$ co-alignment offsets $(\Delta Y)$, relative to $1700 \AA$ image positions, versus time (minutes measured relative to 5 June 2012 23:00:00 UT) shown as a function of AIA telescope number $(1,2$, and 4 only; Lemen et al., 2012) and smoothed over $\approx 30 \mathrm{~min}$ for visual clarity. Pre-processing techniques of MP, SC and CutOut are represented by red pluses, blue $\mathrm{x}$-signs, and gold triangles on each plot, respectively. 
Telescope \#3

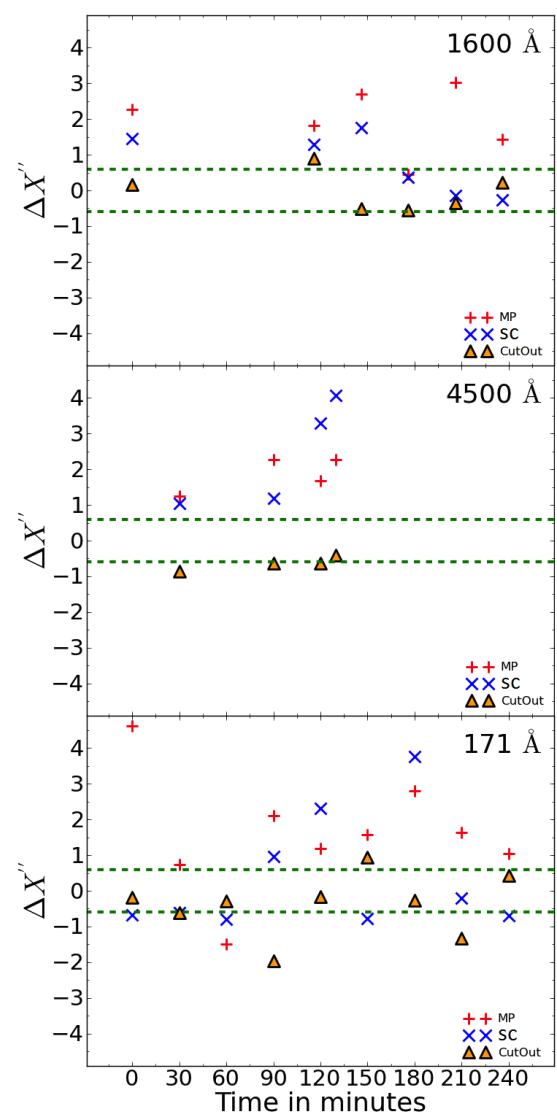

Telescope \#3

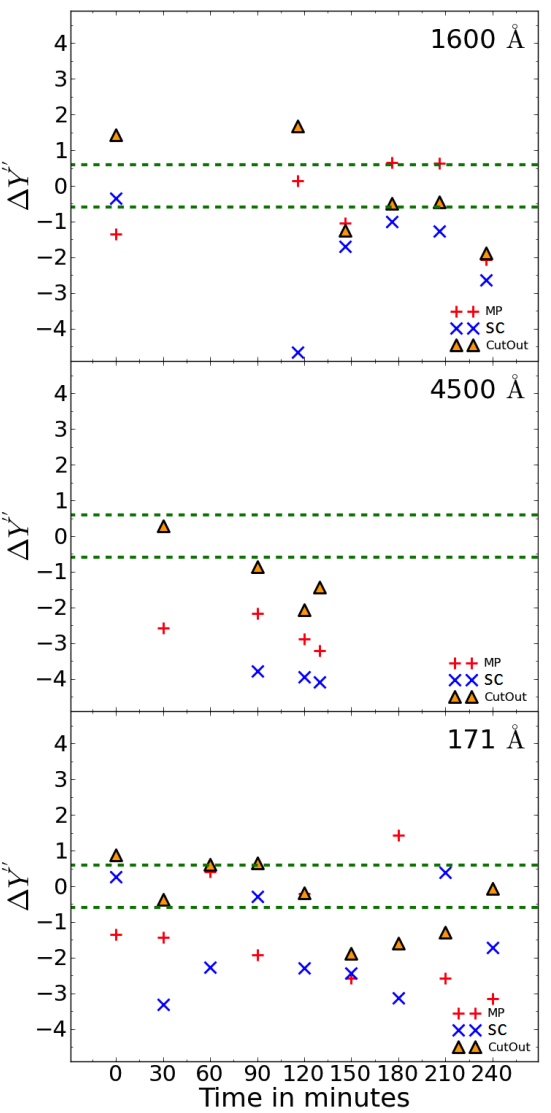

Figure 9. Solar $x$ and $y$ co-alignment offsets $(\Delta X$ and $\Delta Y$ in left and right columns, respectively), relative to $1700 \AA$ image positions, versus time (minutes measured relative to 5 June 2012 23:00:00 UT) shown for AIA telescope number 3 (Lemen et al., 2012) and smoothed over $\approx 30 \mathrm{~min}$ for visual clarity. Pre-processing techniques of MP, SC and CutOut are represented by red pluses, blue x-signs, and gold triangles on each plot, respectively.

in the $x$ and $y$ directions, respectively, independent of the reference band.

\section{Discussion}

Using an extended three year study, a characterization of AIA (1700 $\AA$ ) and HMI's (LOS magnetograms) pointing variations, at sub-weekly time-scales, was performed. Mis-alignments of the two instruments were consistent with previous reports indicating no better than single pixel accuracy exists in the master pointing data (Del Zanna, O'Dwyer, and Mason, 2011; Brooks, Warren, and Ugarte-Urra, 2012 Shine et al., 2011). Pointing variations of the various AIA passbands were determined to be $\lesssim 1.18^{\prime \prime}$ on a daily scale using the recent June 
Table 3. Typical co-alignment offsets, in arcsec, (averaged over 7 AIA passbands) measured relative to the waveband listed in the far left column's image positions, for pre-processing under CutOut assumptions only.

\begin{tabular}{ccccc} 
Band $\AA$ & $\left\langle X_{C O}\right\rangle$ & $\sigma_{S E M}^{X}$ & $\left\langle Y_{C O}\right\rangle$ & $\sigma_{S E M}^{Y}$ \\
\hline 131 & 0.95 & 1.19 & 1.24 & 1.56 \\
171 & 0.98 & 1.27 & 1.14 & 1.39 \\
193 & 0.96 & 1.21 & 1.19 & 1.47 \\
211 & 1.06 & 1.31 & 1.27 & 1.63 \\
304 & 1.31 & 1.62 & 1.41 & 1.72 \\
335 & 0.97 & 1.22 & 1.56 & 1.89 \\
1600 & 1.73 & 2.32 & 1.08 & 1.32 \\
\hline
\end{tabular}

2012 Venus transit, and as such were consistent with the instrument team's expectations $\left(\lesssim 1.2^{\prime \prime} ;\right.$ Shine et al. 2011$)$

Two notable artifacts were identified in the long-term pointing data (Figure 2p. The first was a significant shift in AIA's pointing between December 2011 and January 2012, while the second was annual sinusoidal fluctuations in both HMI and AIA's master pointings (Figure 21). The final artifact was daily variations found in the master pointings of AIA passbands (Figures 7, 8, and 9). Below we provide discussions on the causes of these two artifacts as well as how they relate to the information obtained in this article.

The AIA $1700 \AA$ pointing shift (Figure 2 is due to adjustments made to telescope 3's thermal control parameters to increase the instrument stability (Boerner 2013, private communication). The heater adjustment occurred on 18 January 2012 and was also performed on telescopes 1 and 2. These types of internal updates have been performed at various times in the mission, and in each case updates are made to master pointings to compensate for the resultant shifts.

The seasonal drift in telescope pointings is likely caused by thermal flexing of the instrument. This flexing is considered to occur between the science telescope (i.e., makes passband images), and the guide telescope which drives the image stabilization system (Boerner 2013, private communication). Changes in energy flux due to variations of SDO's distance to the Sun during its yearly orbit, with peak changes correlating with Earth's aphelion and perihelion, are suggested as the origin of the flexing itself. Furthermore, SDO's geosynchronous orbit likely generates these same effects on a daily basis, thereby causing similar daily variations in the master pointings. Temperature variations are believed to be the cause of daily pointing variations $\left(\approx 0.6^{\prime \prime}-1.2^{\prime \prime}\right.$; Shine et al. 2011$)$, to which our results are consistent (Table 3).

AIA and HMI long-term pointing variations in the solar $x$ direction, $\approx 0.26^{\prime \prime}$, indicates the master pointing accuracy is jitter limited at time-scales below their characterization. This conclusion is further supported by the distribution (i.e., random scatter) of our measured $x$ pointings to those of the instrument teams (Figure 22. It is hypothesized that the consistent nature of the $y$ offsets, between 
limb and correlation derived pointings, may be an indication of expected satellite flexing or even a payload shift. Finally, based on minimal variations of correlated offsets between AIA and HMI we hypothesize the thermal jitter affecting HMI is similar in scale to AIA's.

\section{Conclusions}

Our study has provided the first characterization of internal pointing errors occurring across SDO's AIA and HMI instruments using cross-correlation techniques at time scales below the team's master pointing data. Long-term correlation offsets provided an estimate of pointing jitter affecting the AIA and HMI instruments. Our solar $x$ pointing variations $\left(\approx 0.3^{\prime \prime}\right)$, between limb and correlation techniques, are within the limits of thermal jitter in the AIA instrument (Lemen et al., 2012, Aschwanden et al., 2013), while those of the solar $y$ direction $\left(\approx 0.5^{\prime \prime}\right)$ are consistent with expected variations (Shine et al., 2011). Thermal jitter, as previously highlighted, occurs from thermal bending between the science and guide telescopes as a result of orbit induced temperature variations that occur on yearly and daily time scales. Minimal variations between limb and correlation derived pointings indicate that master pointing data achieve single pixel accuracy on sub-weekly time-scales. Long-term instrument pointing variations between HMI and AIA also suggest that thermal jitter affecting HMI is similar in scale to that of AIA.

From the June 2012 Venus transit, daily pointing variations in all AIA passbands were presented. Our results are consistent with the instrument team's expectations of daily pointing shifts of $1-2$ pixels (Shine et al., 2011). The typical co-alignment offsets were $\left\langle\Delta X_{C O}\right\rangle=1.10^{\prime \prime}$ and $\left\langle\Delta Y_{C O}\right\rangle=1.25^{\prime \prime}$, when aligned to HMI's nominal image center. Finally, these co-alignment accuracies are consistent with previously reported mis-alignments of $\approx 1^{\prime \prime}$ in AIA passbands (Del Zanna, O'Dwyer, and Mason, 2011; Brooks, Warren, and Ugarte-Urra, 2012), and expected variations in master pointing data (Shine et al., 2011).

\section{Acknowledgements}

The authors are very grateful for discussions and comments from P. Boerner and to R. Shine for providing us with extensive AIA pointing data, both from the AIA team. The authors are very grateful for the referee's constructive comments and suggestions on this article. This research was supported by the National Aeronautics and Space Administration (NASA) grant NNX-07AT01G. N. B. Orange was also supported by the Florida Space Grant Consortium, a NASA sponsored program administered by the University of Central Florida, grant NNX-10AM01H. Any opinions, findings, and conclusions or recommendations expressed in this material are those of the author(s) and do not necessarily reflect the views of the NSF or NASA. The final publication is available at http://link.springer.com/article/10.1007\%2Fs11207-013-0441-2. 


\section{References}

Aschwanden, M.J., Boerner, P., Schrijver, C.J., Malanushenko, A.: 2013, Automated temperature and emission measure analysis of coronal loops and active regions observed with the Atmospheric Imaging Assembly on the Solar Dynamics Observatory (SDO/AIA). Solar Phys. 283, 5-30. doi $10.1007 /$ s11207-011-9876-5

Boerner, P., Edwards, C., Lemen, J., Rausch, A., Schrijver, C., Shine, R., Shing, L., Stern, R., Tarbell, T., Title, A., Wolfson, C.J., Soufli, R., Spiller, E., Gullikson, E., McKenzie, D., Windt, D., Golub, L., Podgorski, W., Testa, P., Weber, M.: 2012, Initial calibration of the Atmospheric Imaging Assembly (AIA) on the Solar Dynamics Observatory (SDO). Solar Phys. 275, 41-66. doi 10.1007/s11207-011-9804-8

Brooks, D.H., Warren, H.P., Ugarte-Urra, I.: 2012, Solar Coronal Loops Resolved by Hinode and the Solar Dynamics Observatory. Astrophys. J. Lett. 755, L33. doi $10.1088 / 2041-$ 8205/755/2/L33

Del Zanna, G., O'Dwyer, B., Mason, H.E.: 2011, SDO AIA and Hinode EIS observations of "warm" loops. Astron. Astrophys. 535, A46. doi 10.1051/0004-6361/201117470

Kamio, S., Hara, H., Watanabe, T., Fredvik, T., Hansteen, V.H.: 2010, Modeling of EIS spectrum drift from instrumental temperatures. Solar Phys. 266, 209-223. doi $10.1007 /$ s11207010-9603-7

Lemen, J.R., Title, A.M., Akin, D.J., Boerner, P.F., Chou, C., Drake, J.F., Duncan, D.W., Edwards, C.G., Friedlaender, F.M., Heyman, G.F., Hurlburt, N.E., Katz, N.L., Kushner, G.D., Levay, M., Lindgren, R.W., Mathur, D.P., McFeaters, E.L., Mitchell, S., Rehse, R.A., Schrijver, C.J., Springer, L.A., Stern, R.A., Tarbell, T.D., Wuelser, J.-P., Wolfson, C.J., Yanari, C., Bookbinder, J.A., Cheimets, P.N., Caldwell, D., Deluca, E.E., Gates, R., Golub, L., Park, S., Podgorski, W.A., Bush, R.I., Scherrer, P.H., Gummin, M.A., Smith, P., Auker, G., Jerram, P., Pool, P., Soufli, R., Windt, D.L., Beardsley, S., Clapp, M., Lang, J., Waltham, N.: 2012, The Atmospheric Imaging Assembly (AIA) on the Solar Dynamics Observatory (SDO). Solar Phys. 275, 17-40. doi 10.1007/s11207-011-9776-8.

Odenwald, S.: 2012, The mathematics of the transit of Venus. http://sunearthday.nasa.gov/ 2012/articles/ttt_75.php

Poduval, B., DeForest, C.E., Schmelz, J.T., Pathak, S.: 2013, Point-spread functions for the extreme-ultraviolet channels of SDO/AIA telescopes. Astrophys. J. 765, 144. doi $10.1088 / 0004-637 \mathrm{X} / 765 / 2 / 144$

Sarro, L.M., Berihuete, A.: 2011, Statistical techniques for the detection and analysis of solar explosive events. Astron. Astrophys. 528, A62. doi 10.1051/0004-6361/201014894

Schou, J., Scherrer, P.H., Bush, R.I., Wachter, R., Couvidat, S., Rabello-Soares, M.C., Bogart, R.S., Hoeksema, J.T., Liu, Y., Duvall, T.L., Akin, D.J., Allard, B.A., Miles, J.W., Rairden, R., Shine, R.A., Tarbell, T.D., Title, A.M., Wolfson, C.J., Elmore, D.F., Norton, A.A., Tomczyk, S.: 2012, Design and ground calibration of the Helioseismic and Magnetic Imager (HMI) instrument on the Solar Dynamics Observatory (SDO). Solar Phys. 275, 229-259. doi $10.1007 / \mathrm{s} 11207-011-9842-2$

Shimizu, T., Katsukawa, Y., Matsuzaki, K., Ichimoto, K., Kano, R., Deluca, E.E., Lundquist, L.L., Weber, M., Tarbell, T.D., Shine, R.A., Sôma, M., Tsuneta, S., Sakao, T., Minesugi, K.: 2007, Hinode calibration for precise image co-alignment between SOT and XRT (2006 November-2007 April). Pub. Astron. Soc. Japan 59, 845-852.

Shine, R.A., Wolfson, C., Boerner, P.F., Tarbell, T.D., Nightingale, R.W.: 2011, Monitoring image alignments and flat fields for AIA/SDO data images. In: AAS - Solar Phys. Dvi. Abs. 42, 21.26, 2126. 